\title{
INVERSION OF SURFACE WAVE DATA IN SOURCE MECHANISM STUDIES
}

\author{
JORGE A. MENDIGUREN*
}

\begin{abstract}
A least squares method is applied to invert Rayleigh and Love wave observations in source mechanism studies. The method converges to a unique solution independently of the orientation of the initial trial source. Source depth is found by minimizing errors in repetite applications of the method at different trial depths. Application to real requires a careful weighting of observations when the path structure is not well known. The source representation in terms of seismic moment tensor components allows for a simple analysis of the surface wave data resolving power. Complex spectra of Rayleigh waves render a unique solution. Complex spectra of Love waves admit infinite solutions. If a double couple source is assumed, the Love data may have one or three equally satisfactory solutions. When only absolute value of surface wave spectra is available any solution may be rotated $180^{\circ}$ in azimuth and or all the source tensor components changed in sign.
\end{abstract}

RESUMO Utiliza-se o método de mínimos quadrados para inverter dados de ondas Rayleigh e Love no estudo de mecanismo de foco. $O$ método converge para uma solução única, independentemente da orientação do foco inicial. A profundidade hipocentral é encontrada minimizando-se os erros com aplicações sucessivas do método em diferentes profundidades. É necessária uma cuidadosa ponderação das observações para aplicação em dados reais quando a estrutura do meio não é bem-conhecida. A representação do foco em termos das componentes do tensor-momento permite uma análise simples do poder de resolução dos dados de ondas superficiais. Espectros complexos de ondas Rayleigh dão solução única. Espectros complexos de ondas Love admitem infinitas soluções. Supondo-se um duplo par de forças como foco, os dados de Love podem ter uma ou três soluções igualmente satisfatórias. Quando somente se dispõe de dados do valor absoluto do espectro de ondas superficiais, qualquer solução pode ser rotada $180^{\circ} \mathrm{em}$ azimute, e ou todas as componentes do tensor-momento podem mudar de sinal.

INTRODUCTION The pattern of spectra of surface waves radiated from a seismic source is a rather complicated function of the earth model, source orientation and focal depth. That complexity makes it difficult to guess the right source parameters from a simple visual analysis of the observed radiation pattern. Therefore, when inverting surface data it is common practice to apply a visual trial and error approach, which may turn to be a long and tedious search, discouraging a more wide application of surface wave data in source mechanism studies.

This paper is an attempt to find a more systematic and less time consuming approach. The method finally proposed is based on Gilbert (1970) moment tensor representation of of the earthquake source and it is an extension of Gilbert and Dziewonski (1975) technique to invert freee oscillation data.

In the very frequent case of having only spectral amplitude data available, the method amounts to solve a system of non linear equations by repetitive application of least squares. This inversion can not be done in a single step as for free oscillations or surface wave data when both amplitude and phase spectra are available. It was found that the method provides a fast way to determine the source depth. After the source depth is found, the method converges to a unique solution independently of the starting trial source orientation.

Even though, in practical cases, the method depends on the suitable choice of the elastic and anelastic properties of the earth model. To compensate for departures from

* Departamento de Geociências da Universidade de Brasília, 70000 - Brasília, DF, Brasil 
the real earth a careful weighting of observation should be made before inverting. This weighting is the counter part to the smoothing of spectra applied in the visual trial and error approach. Finally it seems unavoidable to make a visual check at some stages of the inversion to verify if the assumed earth model is compatible with the observed radiation.

By expressing the source parameters in terms of the seismic moment tensor components it is easier to analyse the source parameters resolving power of different type of data, like amplitude, or amplitude and phase of Rayleigh waves, or Loves waves, or their combination.

SURFAGE WAVE RADIATION CORRESPONDING TO EACH SEISMIG MOMENT TENSOR GOMPONENT The seismic moment tensor components corresponding to a unit seismic moment double couple source may be expressed as follows:

$$
\begin{aligned}
& M_{x y}=\frac{1}{2} \sin 2 \varphi \sin 2 d \sin s+\cos 2 \varphi \cos s \sin d \\
& M_{y z}=\sin \varphi \cos d \cos s-\cos \varphi \sin s \cos 2 d ; \\
& M_{z x}=\cos \varphi \cos d \cos s+\sin \varphi \sin s \cos 2 d ; \\
& M_{x x}=-\sin ^{2} \varphi \sin 2 d \sin s-\sin 2 \varphi \cos s \sin d \\
& M_{y y}=-\cos ^{2} \varphi \sin 2 d \sin s+\sin 2 \varphi \cos s \sin d \\
& M_{z z}=\sin 2 d \sin s
\end{aligned}
$$

where $\varphi, d$ and $s$ are the strike, dip and slip direction of the fault associated with the double couple. The sign convention for $\varphi, d$ and $s$ are indicated in Fig. 1. Given the symmetry of the seismic moment tensor, it is understood that $M_{i j}=M_{j i}$.

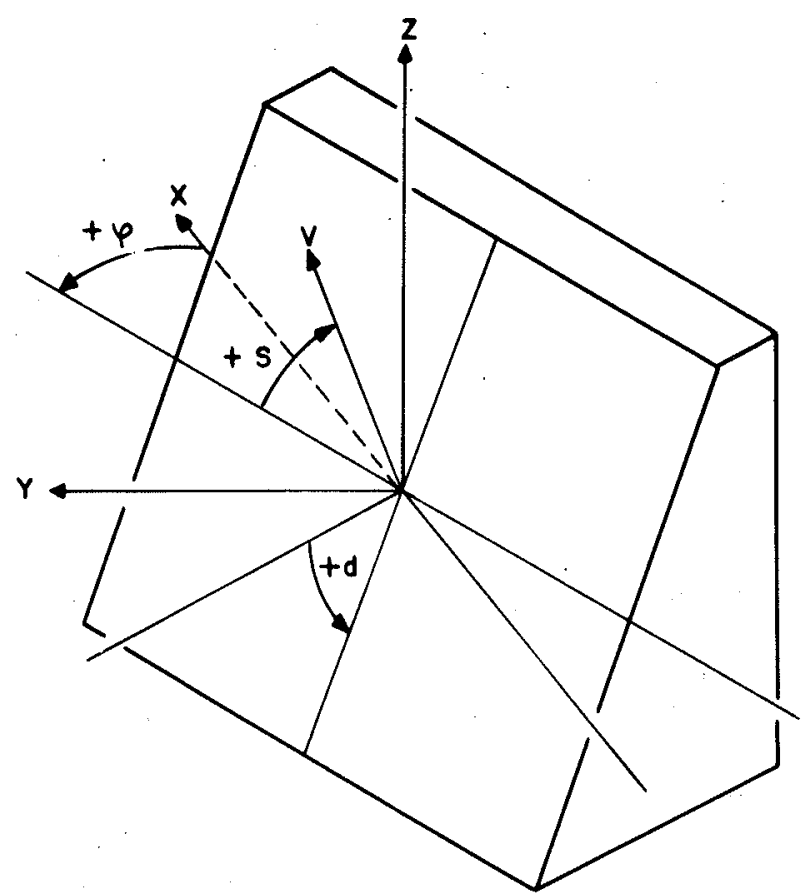

Figure 1 - Lower block of the fault system in relation to the coordenate system $x, y, z$. The $z$ axis points upwards. The direction of fault strike $\varphi$ is measured counterclockwise from $x$. The fault dip angle $d$ is measured positively downward from the horizontal $x y$ plane. $V$ is the slip vector indicating the direction of motion of the upper block over the fault surface. The slip angle $s$ is measured positively clockwise from the strike direction, on the fault plane 
Expressions for the spectral density of the surface wave radiation corresponding to each component of the seismic moment tensor may be derived from Saito (1967). They are the following.

where

$$
\begin{aligned}
\left(F_{y x}^{L}+F_{x y}^{L}\right) & =A^{L} \cos 2 \phi e^{-i \pi / 4} \\
\left(F_{y z}^{L}+F_{z y}^{L}\right) & =-B^{L} \cos \phi e^{i \pi / 4} ; \\
\left(F_{z x}^{L}+F_{x z}^{L}\right) & =B^{L} \sin \phi e^{i \pi / 4} ; \\
F_{x x}^{L} & =-\frac{A^{L}}{2} \sin 2 \phi e^{-i \pi / 4} \\
F_{y y}^{L} & =\frac{A^{L}}{2} \sin 2 \phi e^{-i \pi / 4} \\
F_{z z}^{L} & =0 \\
\left(F_{x y}^{R}+F_{y x}^{R}\right) & =A^{R} \sin 2 \phi e^{i \pi / 4} ; \\
\left(F_{y z}^{R}+F_{z y}^{R}\right) & =B^{R} \sin \phi e^{-i \pi / 4} ; \\
\left(F_{z x}^{R}+F_{x z}^{R}\right) & =B^{R} \cos \phi e^{-i \pi / 4} \\
F_{x x}^{R} & =\frac{A^{R}}{2}(1+\cos 2 \phi) e^{i \pi / 4} \\
F_{y y}^{R} & =\frac{A^{R}}{2}(1-\cos 2 \phi) e^{i \pi / 4} \\
F_{z z}^{R} & =C^{R} e^{i \pi / 4}
\end{aligned}
$$

$$
\begin{aligned}
& A^{L}=\frac{\bar{y}_{1}^{L}(z) \bar{y}_{1}^{L}(0)}{4 C^{2} U I_{1}^{L}} \sqrt{\frac{2}{\pi k r}} e^{-i k r} ; \\
& B^{L}=\frac{\bar{y}_{2}^{L}(z) \bar{y}_{1}^{L}(0)}{4 \mu \omega C U I_{1}^{L}} \sqrt{\frac{2}{\pi k r}} e^{-i k r} ; \\
& A^{R}=\frac{\bar{y}_{3}^{R}(z) \bar{y}_{1}^{R}(0)}{4 C^{2} U I_{1}^{R}} \sqrt{\frac{2}{\pi k r}} e^{-i k r} ; \\
& B^{R}=\frac{\bar{y}_{4}^{R}(z) \bar{y}_{1}^{R}(0)}{4 \mu \omega C U I_{1}^{R}} \sqrt{\frac{2}{\pi k r}} e^{-i k r} ; \\
& C^{R}=\frac{-\bar{y}_{1}^{R}(0)}{4 \omega C U I_{1}^{R}} \sqrt{\frac{2}{\pi k r}} e^{-i k r}\left[\frac{\bar{y}_{2}^{R}(z)}{\lambda+2 \mu}+\frac{\lambda k \bar{y}_{3}^{R}(z)}{\lambda+2 \mu}\right] .
\end{aligned}
$$

Symbols are those used in Saito (1967). Supercripts $R$ and $L$ indicate Rayleigh and Love wave excitation, while the pair of subscripts like $x x, x y$, etc. identify the exciting components of the source seismic moment tensor. The spectral density $F$ corresponds to displacement on the earth surface for each unit component of seismic moment tensor. A step function is assumed as source time function.

Expressions (2) are based on the following sign convention

$$
F(\omega)=\int_{-\infty}^{\infty} f(t) e^{-i \omega t} d t,
$$

$\mu$ and $\lambda$ are the earth model elastic constants at the source depth. Then, the displacement spectra for an arbitrary source is given by

$$
F=\sum_{i} \sum_{j} M_{i j} F_{i j}
$$


Now, introducing (2) and (3) in (4) we obtain

$$
\begin{aligned}
& F^{L}=A^{L}\left(M_{x y} \cos 2 \phi+\left(M_{y y}-M_{x x}\right) \frac{1}{2} \sin 2 \phi\right) e^{-i \pi / 4}+ \\
& B^{L}\left(-M_{y z} \cos \phi+M_{z x} \sin \phi\right) e^{i \pi / 4}, \\
& F^{R}=A^{R}\left(M_{x y} \sin 2 \phi-\left(M_{y y}-M_{x x}\right) \frac{1}{2} \cos 2 \phi\right) e^{i \pi / 4}+ \\
& +B^{R}\left(M_{y z} \sin \phi+M_{z x} \cos \phi\right) e^{-i \pi / 4}+ \\
& +\left(C^{R} M_{z z}+\frac{A^{R}}{2}\left(M_{y y}+M_{x x}\right)\right) e^{i \pi / 4} \\
& \left|F^{L}\right|=\left[A^{L^{2}}\left(M_{x y} \cos 2 \phi+\left(M_{y y}-M_{x x}\right) \frac{1}{2} \sin 2 \phi\right)^{2}+\right. \\
& \left.+B^{L^{2}}\left(-M_{y z} \cos \phi+M_{z x} \sin \phi\right)^{2}\right]^{1 / 2} \text {, } \\
& \left|F^{R}\right|=\left[A ^ { R ^ { 2 } } \left(M_{x y} \sin 2 \phi-\left(M_{y y}-M_{x x}\right) \frac{1}{2} \cos 2 \phi+M_{z z} C^{R}+\right.\right. \\
& \left.\left.+\left(M_{y y}+M_{x x}\right) \frac{A^{R}}{2}\right)^{2}+B^{R^{2}}\left(M_{y z} \sin \phi+M_{z x} \cos \phi\right)^{2}\right]^{1 / 2} .
\end{aligned}
$$

Expressions (5) where checked comparing explicit expressions for double couple sources given in Saito (1967) with those obtained introducing (1) in (5).

INVERSION OF SURFAGE WAVE SPECTRA TO RECOVER THE SOURGE MOMENT TENSOR The problem of deriving the source moment tensor components from observations of Love and or Rayleigh waves when absolute value or complex displacement spectra is available will be considered next.

When complex spectra $F^{L}$ is available, only two observations are required to solve for $M_{x y}, M_{y x}, M_{z x}$ and $\left(M_{y y}-M_{x x}\right)$. As the component $M_{z z}$ or a pair $M_{x x}=M_{y y}$ does not radiate Love waves we can not solve for all components of the seismic moment tensor unless some restrictions are imposed.

The absence of an isotropic component would be implied by the restriction $\Sigma M_{i i}=0$. The assumption that the intermediate principal component is zero, as required for a planar shear source, would be equivalent to set the determinant of the moment matrix equal to zero. Then the unknown components $M_{j j}$ are found solving the set of equations

$$
\begin{aligned}
& M_{x x}+M_{y y}+M_{z z}=0 \\
& M_{y y}-M_{x x}=C \\
& M_{x x} M_{y y} M_{z z}+2 M_{x y} M_{y z} M_{z x}-M_{x z} M_{y y} M_{x z}-M_{x y} M_{y x} M_{z z}-M_{x x} M_{y z} M_{z y}=0,
\end{aligned}
$$

where $C$ and $M_{i j}$ are observed values.

The system (6) is reduced by substitution to a third degree equation

$$
\begin{aligned}
M_{x x}^{3} & +\frac{3}{2} C M_{x x}^{2}+\frac{1}{2}\left(C^{2}+M_{x z}^{2}-2 M_{x y}^{2}+M_{y z}^{2}\right) M_{x x}+ \\
& +\frac{1}{2}\left(-2 M_{x y} M_{y z} M_{z x}+M_{x z}^{2} C-M_{x y}^{2} C\right)=0
\end{aligned}
$$

In general, the equation (7) will have three solutions, being one or all them real. In order to find out when we should expect to find one or a set of three real solutions, the descriminant of (7), Rey Pastor et al. (1961), was numerically computed for different orientations of a planar shear field. It was found that when $|d|<35^{\circ}$ the system (6) has only one real solution, while when $|d|>35^{\circ}$, in most cases, there are three real solutions. The complete situation is shown in Fig. 2.

Therefore when $F^{L}$ data is available and a planar shear source is assumed, we obtain one or three solutions satisfying the observed data. Fig. 3 shows one example of three different double couple sources with the same $F^{L}$ radiation pattern. 


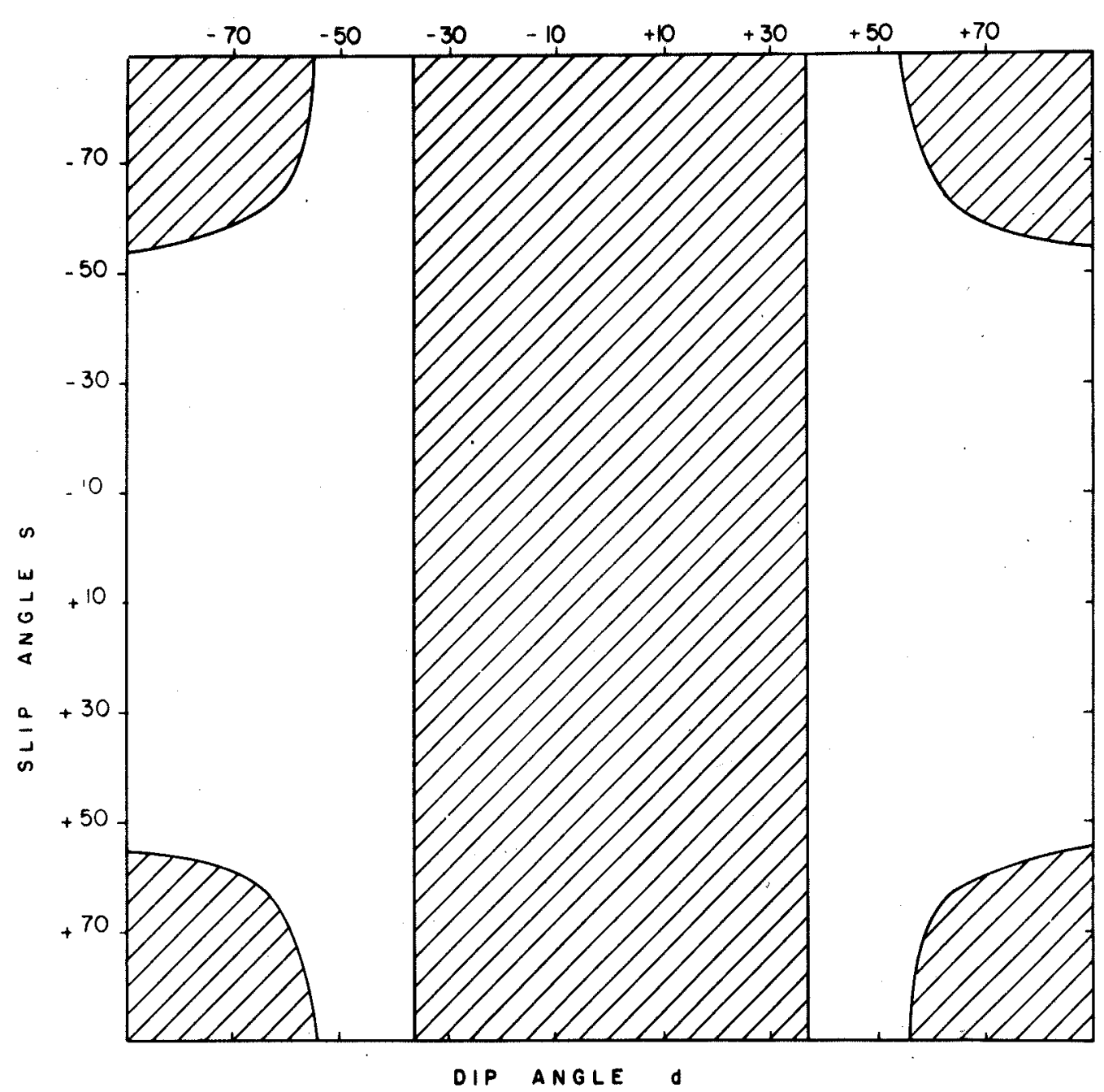

Figure 2 - Shadow zones indicate combinations of dip $d$ and slip $s$ for which there is a one to one correspondence between source orientation and spectra of radiated Love wave. Glear zones correspond to combinations of $s$ and $d$ where three differents $s, d$ pairs render equal Love wave radiation

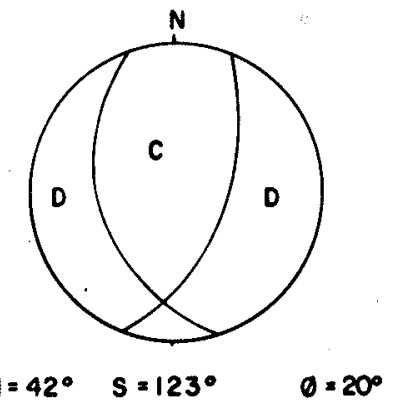

$M=1.42$.

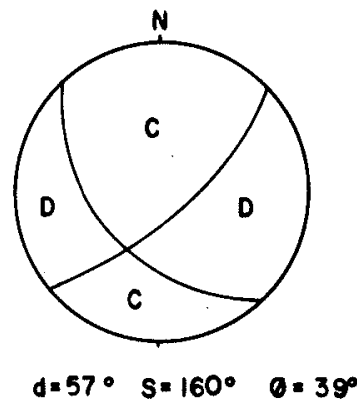

$M=1.00$

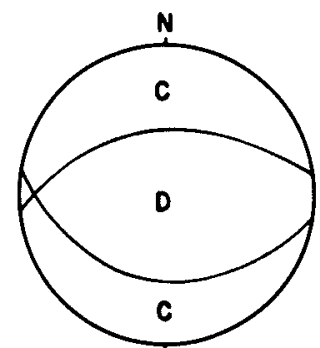

d $=36^{\circ} 5=-105^{\circ} 0=79^{\circ}$

$M=1.63$

Figure 3 - Three different source mechanisms rendering equal spectra of Love waves 
It should be noted that their seismic moment tensor is different in orientation and strength.

The three real solutions of (6) are not a simple interchange of principal axis of stress or a $\pi$ azimuth rotation of the whole moment tensor.

If only $\left|F^{L}\right|$ data is available we may solve for $M_{x y}, M_{y z}, M_{z x}$ and $\left(M_{y y}-M_{x b}\right)$ as before. A minimum of four observations at different stations, or ar four different periods for the same station are required. But now, it is possible to change the sign of all components and to obtain the same radiation $\left|F^{L}\right|$. This is seen by inspection of $\left|F^{L}\right|$ in (5), and it is equivalent to interchange the tension and pressure axis of the modal plane solution. It is also possible to change $\phi$ by $\phi+\pi$ without altering $\left|F^{L}\right|$. This is equivalent to $180^{\circ}$ azimuth rotation of the modal plane solution. Therefore we may have four or twelve different solutions satisfying $\left|F^{L}\right|$ data.

In case of Rayleigh wave data we need at least three observations of the complex spectra $F^{R}$ to solve for $M_{x y}, M_{y z}, M_{z x},\left(M_{y y}-M_{x x}\right)$ and $\left(M_{z z}+\left(M_{x x}+M_{y y}\right) \frac{1}{2} A^{R} / C^{R}\right)$. As pointed out by Mendiguren and Aki (in press), we may solve for $M_{z z}, M_{x x}$ and $M_{y y}$ only if we have observations at different periods for which $A^{R} / 2 C^{R}$ is significantly different. Otherwise we may allways exchange between $M_{z z}$ and $\left(M_{x x}+M_{y y}\right)$ and still satisfy the data. Fig. 4 shows the value of $A^{R} / 2 C^{R}$ as a function of frequency and focal depth for an oceanic model. It shows that, for example, for a $11 \mathrm{~km}$ depth shock the resolving power of Rayleigh data is larger than for a $40 \mathrm{~km}$ depth case.

Assuming the absence of an isotropic source, $\Sigma M_{i i}=0$, the tensor components are found uniquely even with $F^{R}$ data corresponding to the same frequency. If we have only $\left|F^{R}\right|$ observations available there will be four possible solutions, corresponding to combinations of a $\pi$ azimuth rotation of the whole moment tensor with an interchange of pressure and tensior axis.

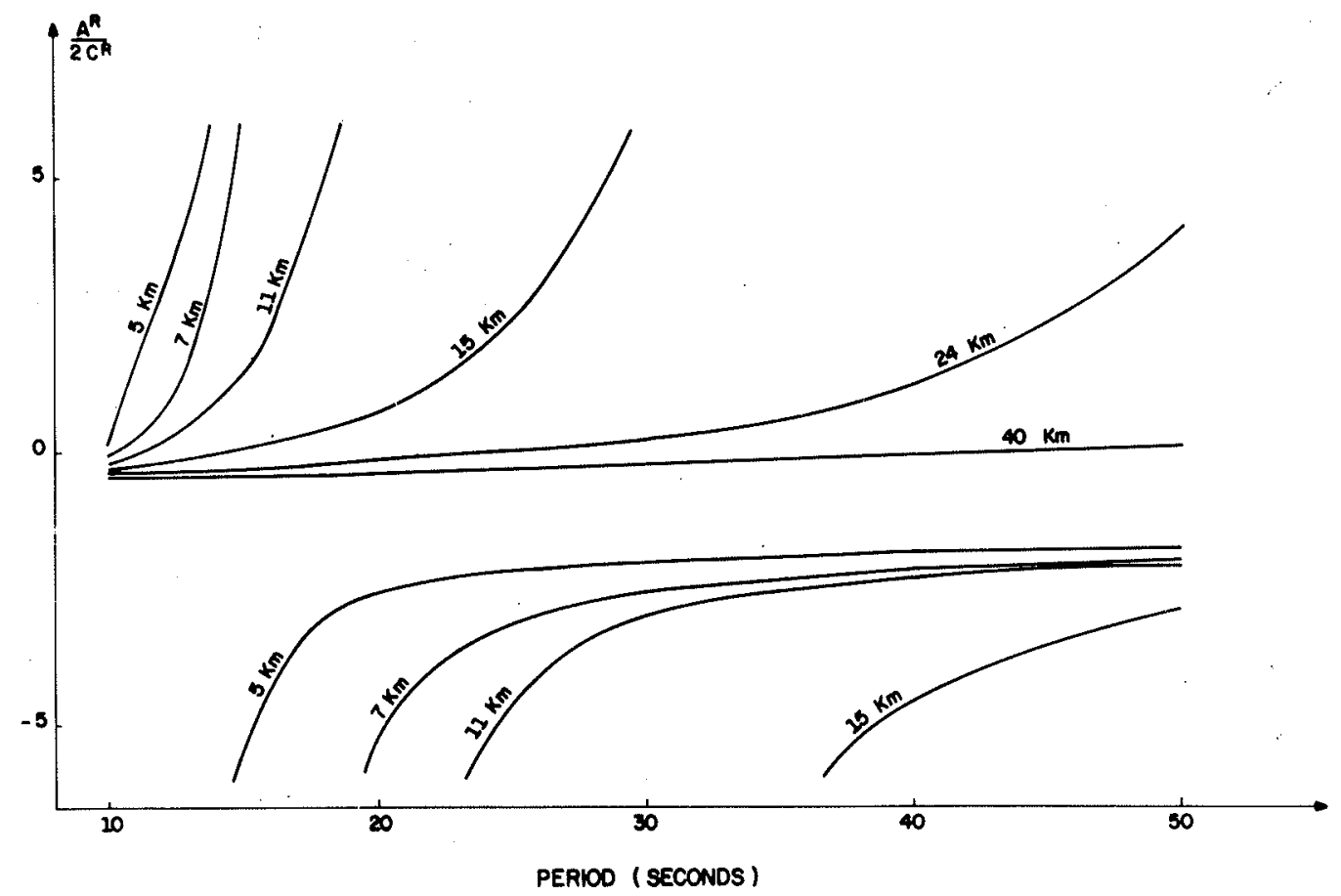

Figure 4 - Coefficient $A^{R} / 2 C^{R}$ for different periods of Rayleigh waves and different source depths 
LEAST SQUARE PROCEDURE TO INVERT SURFACE WAVE DATA As shown in (5) the real and imaginary parts of the complex spectra $F^{L}$ and $F^{R}$ are linear functions of the seismic moment tensor components. When the number of observations is larger than the number of unknowns we may apply a least square method and solve for the tensor components in one single step. Unfortunately the complex spectra $F^{L}$ and $F^{R}$ are rarely available, specially at periods less than 20 seconds where the phase velocity is not always precisely known for different paths. When only the absolute values of the spectra $\left|F^{L}\right|$ or $\left|F^{R}\right|$ are known, a single step least square procedure can not be applied because $\left|F^{L}\right|$ and $F^{R}$ are not linear functions of the tensor components. We may start from a trial tensor and reach a solution by stepwise applications of least squares till satisfying a given convergence criterium.

In order to test the practical merits of this approach we proceeded as follows. The values of $\left|F^{\mathbf{L}}\right|$ and $\left|F^{R}\right|$ for $\mathbf{8}$ stations randomly distributed around the epicenter were computed for a source at $9.0 \mathrm{~km}$ depth, $\phi=0, d=48^{\circ}$ and $s=-68^{\circ}$ in an oceanic structure. Then, those computed amplitudes $\left|F^{L}\right|$ and $\left|F^{R}\right|$, between 10 and 50 second period, were used as input in the least square inversion scheme. The method was applied separately at different focal depths, and in each case the initial trial tensor was allowed to span all possible space orientations. It was found that for each depth the method converged to the same solution independently of the orientation of the initial trial tensor. Fig. 5 shows the sum of the resulting squared errors for the solutions at each depth. It has a minima at the source depth $9.0 \mathrm{~km}$.

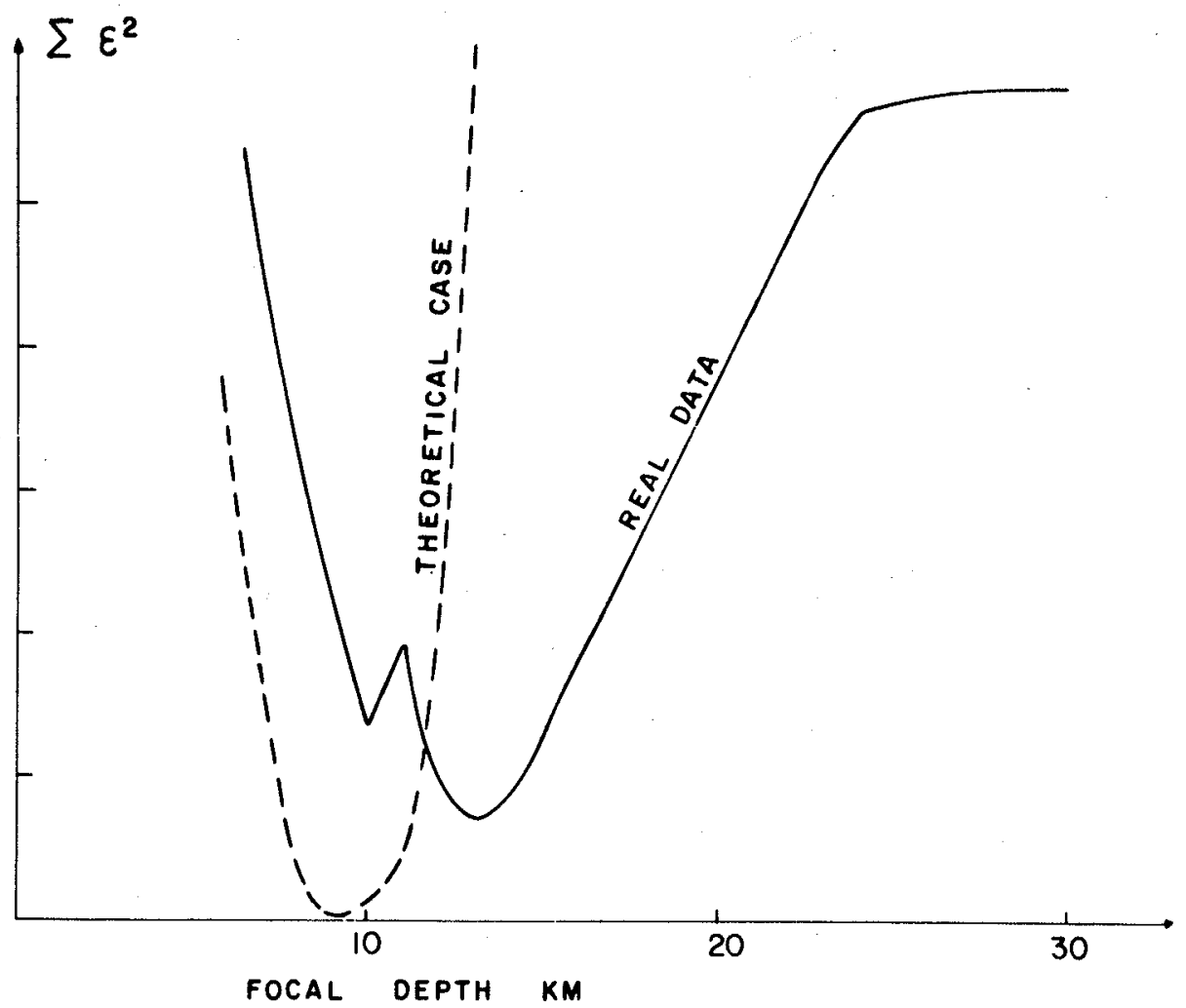

Figure 5 - Sum of squared errors resulting when applying the least squares method for different focal depths for theoretical and real data 
In order to test the method with real data we inverted Love and Rayleigh wave absolute amplitude spectra observed for an oceanic shock (Mendiguren, 1971). The condition $\Sigma M_{i i}=0$ was imposed to the solution. In a first trial all observations were given the same weight and it was found that the solution was incompatible with first motion of $P$ data. This fact pointed to the necessity of carefully weighting the data before inversion. This weighting is equivalent to the subjective smoothing of spectra usually applied when visually comparing observed to theoretical spectra. Smaller weight was given to data at short periods were $F$ is highly dependent on path structure (Weidner, in press). Besides, the ratio of Love to Rayleigh wave amplitude is known to be dependent on the earth structure, and this should be considered when mixing data of both types.

Fig. 5 shows the sum of weighted errors for different depths obtained for the oceanic shock, while Fig. 6 shows the source mechanism obtained with the least square technique

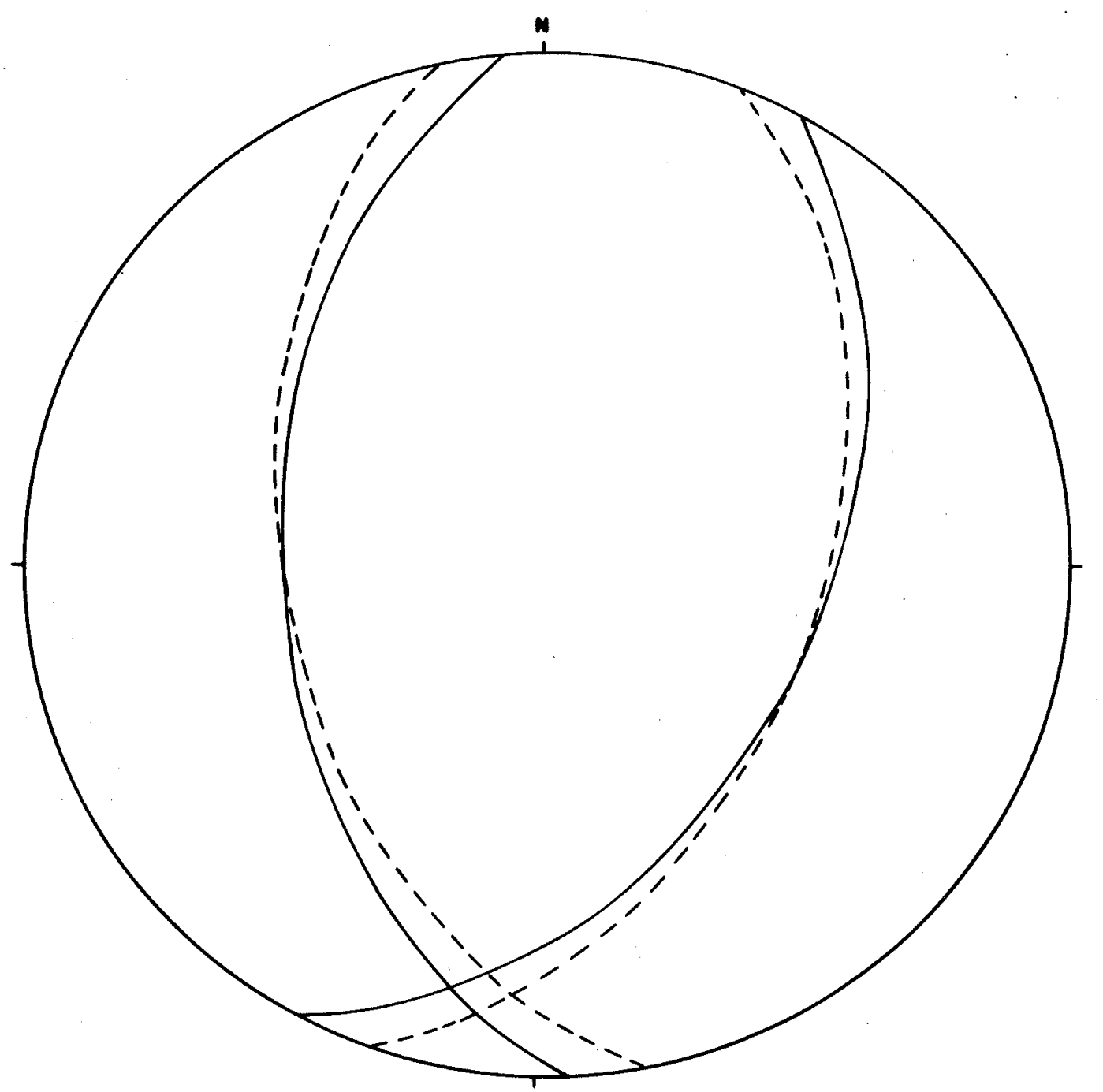

Figure 6 - Source mechanism obtained by the usual visual technique (continous lines) compared to that obtained using the least squares method (dash line) 
compared to the solution formerly obtained by the visual trial and error method. They are pretty close while the source depth was different by $0: 5 \mathrm{~km}$.

The moment tensor solution had a non zero intermediate principal stress. This implies the existence of a compensated dipole (Knopoff and Randall, 1970), beside the double couple source. If we define the moment of the compensate dipole as the moment of its main dipole, and assume that the main dipole coincides with the principal axis of stress, then the observed moment tensor would include a compensated dipole of moment 0.1 of the double couple seismic moment. If we postulate that a compensate dipole component does not exist in the real source physical process, therefore, the value of the intermedium principal stress may be used as a measured of the solution quality.

Those results indicate that the least square method applied to solve for the component of the moment tensor is reliable and much faster than the visual trial and error approach. Nevertheless, lateral heterogeneities and departures from the assumed $Q$ model may produce distortions in the observed spectra which could lead to large errors in the source parameters derived in a blind application of the least square method. Those distortions may only be noted by visual analysis of radiated spectra. Therefore the least square method is more efficient when the path structure is reasonably well known. In case of complicated or unknown path structure some graphical checking during the inversion seems to be unavoidable.

Note: It was pointed out to the author that Cowan (1976) has also published explicit expressions for the excitation of Love and Rayleigh waves as function of the moment tensor components.

Acknowledgements This paper was much improved after critical reviewing by $\mathrm{K}$. Aki and R. Madariaga. The author is grateful to them. The author is also indebted to J. A. Tirao for his help in the mathematics of this paper. This study was partially supported by a grant from Conselho Nacional de Desenvolvimento Científico e Tecnológico of Brazil.

\section{REFERENCES}

COWAN, D. W. - 1971 - Moment tensor representation of surface wave sources. Geophys. J. R. Astr. Soc., 44: 595-599

GILBERT, F. - 1970 - Excitation of the normal modes of the earth by earthquake source. Geophys. J. R. Astr. Soc., 22: 223-226

GILBERT, F. and DZIEWONSKI, A. M. - 1975 - An application of normal mode theory to the retrieval of structural parameters and source mechanisms from seismic spectra. Phil. Trans. Royal Soc. London, 278, A., 1280: 187-269

KNOPOFF, L. and RANDALL, J. - 1970 - The compensated linear-vector dipole: A possible mechanism for deep earthquake. J. Geophys. Res., 75: $4957-4963$

MENDIGUREN, J. A. - 1971 - Focal mechanism of a shock in the middle of Nazca plate. J. Geophys. Res., 76: $3861-3879$

MENDIGUREN, J. A. and AKI, K. (in press) Source mechanism of the deep Colombian earthquake of July 31, 1970 from free oscillation data. Geophys. J. R. Astr. Soc.

REY PASTOR J., PI GALLEJA P., and TREJO, G. A. - 1961 - Análisis Matemático. Ed. Kapelusz S.A., Buenos Aires

SAITO, M. - 1967 - Excitation of free oscillations and surface waves by a point source in a vertically heterogeneous earth. J. Geophys. Res., 72: 3689-3 699

WEIDNER, D. J. (in press) The effect of oceanic sediments on surface wave propagation 